\title{
La actividad administrativa de las regiones en Italia
}

SUM.ARIO : I. Introducción: 1. Antecedentes históticos.-2. Principios generales.-3. Organos de la Región.-4. Materias que pueden ser objeto de la actividad regional. -5 . La actividad legislativa de las Regiones.II. Importancia de la actividad administrativa regional.-1II. Peculiaridades ae la actividad administrativa regional.-IV. Relaciones entre el Estado y la Región: el control estatal sobre los actos regionales.-V. Relaciones entre la Región y las Provincias y Municipios y demás entes locales.-VI. Resultados de la actividad administrativa regional.

1.-La articulación regional del Estado es una vieja aspiración de políticos y juristas italianos $\mathrm{Ya}$ Cavour señalaba el malestar producido por la extensión de las leyes unificadoras piamontesas a las provincias anexionadas y sugería como posible remedio la institución de un ente intermedio entre Provincias y Municipios de un Iado, y Estado de otro; Marco Minguetti, además de ocuparse en teoría del problema, llegó incluso a presentar a la Cámara, en unión de Farini, un proyecto de ley en tal sentido, que fué rechazado por la preocupación de evitar cualquier posible atentado a la unidad (1). No tuvieron mayor éxito los escritos de eminentes estudio sos, como Saredo, ni las numerosas tentativas que se producen al

(1) Vide, sobre el particular, SAREDo: La legge comunale anotatta, vol. 1, págs. 147 y ss. CAIIENDA : La regione nel ordinamento amministrativo italiano, Koma, 1895. Citados por Zanobini : Corso di Diritto Amministrativo, vol. I.II, 5. ${ }^{a}$ ed., Milán, 1955, pág. 122, en nota. 
final de la primera guerra mundial (2). Sólo después de la segunda, la solución afirmativa encuentra favorable acogida (3) al promulgarse la nueva Constitución, cuyo artículo $50^{\circ}$ declara que la República se divide en Regiones, Provincias y Municipios (4).

2.- - Las normas fundamentales acerca de la Región se contienen en el Título V de la Constitución (arts. 114-132), no pudiendo ser modificadas sino por los procedimientos especiales de las leyes constitucionales (art. 138). Dichos artículos se encuentran integrados por los preceptos de la Ley de 10 de febrero de 1953, número 62, sobre la constitución y el funcionamiento de los órganos regionales, no habiendo sido aún promulgada la ley sobre la elección y composición de los Consejos regionales. Por otra parte, cada Región tiene un estatuto que, en armonía con la Constitución y las leyes de la República, establece las normas relativas a la organización interna de la Región (art. 123 de la Constitución). El artículo $1 .^{\circ}$ de la citada Ley de 1953, nfmero 62, especifica que el estatuto regional debe contener normas sobre la organización de las oficinas regionáles, y sobre el funcionamiento del Consejo y de la Junta regionales; sobre las relaciones entre Consejo, Junta y Presidente regionales; sobre la delegación de funciones administrativas de la Región a Provincias, Municipios $u$ otros entes locales, para objeto definido y por tiempo determinado; sobre la eventual institución de nuevas circunscripciones, y sobre los funcionarios de la Región. De esta suerte, como señala Zanobini (5), a diferencia de la uniformidad de ordenamiento que es propia de los. Municipios y Provincias, las - Regiones, con la excepción de. los puntos fundamentales fijados por

(2) Vide Virta: Il Regionalismo, Florencia, 1922. y S. D'Amalo: Autonomia regionale e Stato unitario, en aRivista di Diritto Pubblicon, 1924, 1, pág. 75. Tambiér citados por Zanobini, en el mismo lugar.

(3) Ia coincidencia del planteamiento del problema del regionalismo con los momentos cruciales de la historia constitucional italiana, se presta a múltiples consideraciones, entre otras, la del triple carácter técnico, politico e histórico de dicho problema, como ha sido puesto de manifiesto por Benevenutr : Risultati e prospettive dell'attivitd regionale, en Atti del Primo Convegno di Studi Regionali, Padua, 1955, pág. 473.

(4) Para un estudio acerca de la Región en Italia pueden consultarse : Rizzo: La regione, Roma, 1947; MrELE : La regione, en Comentario sistemático della Costituzione italiana, dirigido por CaLAMANDREI y LEVI, Florencia, 1949 ; VrRGa : La regione, Milán, 1949, y los Atti cit.

(5) Corso cit., vol. III, pág. 178. 
la Constitución y por las leyes de ejecución, tendrán ordenamien. tos en parte diversos, de acuerdo con la distinta extensión, densidad de población, ocupaciones prevalentes e importancia de la Administración en la vida social de cada una de ellas.

Las normas fundamentales contenidas en la Constitución y, en la citada ley de ejecución no se aplican a cinco Regiones, a las que, por razones geográficas o históricas, se concede (art. 116 de la Constitución) un régimen especial. El ordenamiento de tales Regiones-Sicilia, Cerdeña, Trentino-Alto Adigio, Valle de Aosta y Friuli-Venecia Julia-es establecido en todos sus extremos por sus respectivos estatutos, que, dado que constituyen parcialmente las normas de la Constitución, han sido aprobados mediante ley constitucional (6). Dichos estatutos atribuyen a las Regiones. a que se refieren una autonomía superior a la que disfrutan las restantes Regiones.

El artículo 131 establece el número y denominación de las Regiones (7). Isa creación de nuevas Regiones y la fusión de las existentes sólo puede teuer lugar mediante' ley constitucional y con la condición de que la nueva Región renga por lo menos un millón de habitantes, que la fusión sea solicitada por tantos consejeros regionales que representen por lo menos un tercio de las poblaciones interesadas y que sea aprobada con referéndum de la mayoría de dichas poblaciones. En cambio, la segregación de Provincias o Municipios de una Región y la agregación a otra, puede verificarse mediante ley ordinaria, siempre que la soliciten los entes interesados, cídos los Consejos regionales y previo el referéndum de las poblaciones (art. 132).

3.-Los ćrganos fundamentales de la Región son el Consejo, la Junta y el Presidente de la misma.

El Consejo emana el estatuto de la Región y las leyes y reglamentos atribuídos a la potestad legislativa de la misma; propone

(6) El Estatuto de la región siciliana fué aprobado por el D. L. de 15 de maỹo de 1945, núm. 455; los de Cerdeña, Valle de Austa y Trentino-Alto Adigio, dirigido' por sendas leyes constitucionales de 20 de febrero de 1946 , núms. 3,4 y 5 , respectivamente. Tanto éstas disposicioncs como sus normas de actuación pueden consultarse en cl Codice Costituzionale, de Pr.rgolessi, Bo!onia, 1955.

(7) Las regiones comprendicias en el articulo 131, son las siguientes : Piamonte, Valle de Aosta, Lombardía, Trentino-Alto Adigio, Veneto, FriuIi-Venecia Julia, Iiguria, Entilia-Romaña, Toscana, Unibria, Híarcas, Iazio, Abruzzos y Mnlise; Campania, Puglia, Basilicata, Sicilia y Cerdeña. 
proyectos de ley al Parlamento y da parecer en los casos que señalan los artículos 123 y 133 de la Constitución ; aprueba el presupuesto ; delibera acerca de los impuestos regionales, del ordenamiento de las oficinas y de los servicios regionales y de ia institución de entes administrativos dependientes de la Región; aprueba los planes generales relativos a la ejecución de obras públicas y a la organización de servicios páblicos de interés de la Región, y en general delibera sobre cualquier otra materia para la cual la ley requiera la aprobación del Consejo o que sea sometida a la votación del Consejo mismo por la Junta (art. 22 de la Ley de 1953).

El Consejo es elegido por la población de la Región dividida en colegios; el sistema electoral, el número de personas que hayan de componer el Consejo y demás extremos relativos a las elecciones deberán ser regulados por una futura ley ordinaria, la inexistencia de la cual es la causa determinante de que, por ahora, la estructuración regional del Estado sólo se haya llevado a la práctica en las Regiones que disfrutan de un régimen especial. Dichas Regiones tieṇen también un Consejo, que en Sicilia se Hama Asamblea regional, y en el.Valle de Aosta., Consejo del Valle.

La Junta es el 6rgano ejecutivo de la Región. Se compone del Presidente y de asesores, efectivos y suplentes, cuyo numero oscila entre seis y dos, reșpectivamente, y doce y cuatro, segun la población de la Región de que se trate. Los asesores son elegidos por el Consejo en' su propio seno, no pudiendo formar parte de la Junta coritemporáneamente ascendientes $\mathrm{y}$ descendientes, hermanos, cónyuges, afines en el primer grado, adoptante y adoptado.

Además de cuidar de la ejecución de las leyes y de las decisiones del Consejo, la Junta ad́ministra el patrimonio de la Región, elabora el presupuesto y controla la gestión de servicios ṕablicos regiovales prestados por entes especiales. En situaciones de urgencia puede dictar actos administrativos de competencia del Consejo.

En las regiones con régimen especial, la posición de la Junta es semejante a la expuesta. En Sicilia y en Trentino-Alto Adigio corresponde a este órgano la potestad reglamentaria, que, como se sabe, normalmente pertenece al Consejo.

El Presidente de la Junta es elegido entre los consejeros. A diferencia de lo que ocurre en el Municipio, la Región tiene dos Presidentes, el del Consejo y el de la Junta. Las funciones del primero se reducen a la dirección de las sesiones del Consejo. En cambio, el Presidente de la Junta, además de presidir los trabajos de ésta, pro- 
mulga las normas jurídicas deliberadas por el Consejo y ostenta la representación exterior y en juicio de la misma.

4.-El artículo 117 de la Constitución enumera una serie de materias que, según se desprende del artículo 118 , pueden ser objeto tanto de la actividad legislativa como de la actividad adminisírativa de las Regiones. Son las siguientes : ordenamiento de las oficinas $y$. de los entes dependientes de la Región ; términos municipales ; policía local, urbana y rural; ferias y mercados; beneficencia pública y asistencia sanitaria; instrucción artística y profesional; museos y bibliotecas de los entes locales; urbanismó; turismo e industria hotelera, tranvías y transportes por carretera de interés regional; obras públicas; aguas minerales y termales; navegación y puertos lacustres; minas; caza y pesca en las aguas internas; agricultura, $y_{\text {; }}$ artesanía. Como ha sido indicado con anterioridad, las Regiones sometidas a un régimen especial gozan de una mayor autonomía.

5.-La actividad legislativa de las Regiones es de tres clases : exclusiva, complementaria e integrativa.

Se entiende por actividad legislativa exclusiva aquella en que poder legislativo de la Región debe ser ejercitado en armonia con la Constitución y con los principios del ordenamiento jurídico y respetando las obligaciones internacionales, los intereses nacionales y las normas fundamentales de las reformas ecouómico-sociales ie la República (8). Se encuentra sólo en las Regiones con autonomía especial.

En el ejercicio de la actividad legislativa concurrente la R'egióir encuentra, además ide los límites fijados en el párrafo anterior, el de los principios establecidos por las leyes del Estado (9).

Finalmente, con la actividad legislativa complementaria, la Región tiende a adaptar a sus particulares exigencias las leyes da la República, emanando normas de integración y actuación (10)

(8) Vide, por ejemplo, el artículo 3 del Estatuto sardo.

(9) Vide artículos 117, 121 y 127 de la Constitución y articulo 4 del Estatuto sardo.

(10) Vide articulo 117 y 121 de la Constitución (párróios último y segundo, respectivamente), y artículo 5 del Estatuto sardo. 
Una rápida ojeada de los principios expuestos acerca de la actividad legislativa de las Regiones bastará para percatar al lector de la importancia de la innovación que se introduce: se reconoce a un ente, dentro del marco del Estado, potestad de emanar normas jurídicas primarias, es decir, normas que poseen la misia eficacia jurídica que las normas ordinarias emanadas por el Estado. Este hecho, unido a la circunstancia de que tanto el artículo 118 de la Constitucion, como los correspondientes artículos de los estatutos de las Regiones especiales, establecen que la Región ejercita las funciones administrativas en las materias para las que ba sido concedida la función legislativa, han ocasionado que la atención de la doctrina se fijase preferentemente en esta última, llegando incluso a considerar la actividad administratîva regional como un apéndice de la legislativa. Postura completamente distinta es la mantenida por Amorth, que recientemente, en trabajos que acreditan su prestigio de exquisito jurista (11), no sólo niega este carácter secundario de la función administrativa, sino que afirma es la función esencial a desarrollar por las Régiones, en consideración a la cual les ha sido concedido el ejercicio de la funcion legislativa. Veamos los argumentos empleados para demostrarlo:

En primer lugar, la fórmula que adoptan tanto la Constitución como los estatutos de las Regiones especiales es reversible, si se considera que la enumeración de las materias en que las Regiones pueden emanar normas legislativas, no es sólo una determinación de la función legislativa regional, sino, sobre todo, una delimitación de la competencia o campo general de actividad de las Regiones. El hecho de que dicha enumeración se haya verificado precisamente con relación a la actividad legislatîva, se explica fácilmente si se tiene en cuenta la novedad que la atribución de la facultad de emanar normas jurídicas primarias supone, que hacía necesario un mayor relieve constitucional que la atribucion de la función administrativa no requería.

(11) L'attivitd amministrativa delle Regioni, en Atti cit., págs. 301, $319 \mathrm{y}$, en particular, el cap. II. 
Pero es que, por otra parte, un análisis de las materias confiadas a la disciplina legislativa de las Regicnes confirma su naturaleza intrínsecamente administrativa, $y$, por tanto, la esencia intrínsecamente administrativa de la actividad de las Regiones mismas. Ello se demuestra también por el hecho de que o se incluyen o so incluían entre las funciones, sin duda administrativas, de los $\mathrm{Mu}$ nicipios o Provincias, o en la competencia administrativa del Estado.

En tercer lugar, la autonomía financiera concedida a las Regiones, a la que tanta importancia se da tanto en. la Constitución como en los estatutos de las Regiones especiales (12), sólo puede explicarșe en relación con el desenvolvimiento de la actividad administrativa, y no con el de la legislativa. Igual puede afirmarse respecto de la existencia, constitucionalmente establecida, de un dominio ptiblico y un patrimonio regional.

El ́́ltimo argumento que Amorth emplea para demostrar la consistencia administrativa de las Regiones es el de la alineación, dispuesta por la Constitución en muchas ocasiones, de la Región junto con los Municipios y Provincias. En efecto: el título quinto de la Constitución se denomina aLas Regiones, las Provincias y los Municipiosn; según el artículo 114, ala República se divide en Regiones, Provincias y Municipios»; la calificación de ente autónomo. se aplica tanto a las Regiones como a las Provincias y Municipios en los artículos 115 y 128 ; finalmente, los tres entes aparecen unidos una vez más en el artículo $5^{\circ}$, relativo a las autonomías locales e inscrito entre los principios fundamentales, donde se declara que la Republica, una e indivisible, reconoce y promueve las autonomías locales, y adapta los principios y los métodos de su legislación a las exigencias de la autonomía y de la descentralización (13).

La atribución a la Región de una naturaleza esencialmente administrativa es rica en consecuencias, entre las que merecen destacarse el carácter necesariamente público de la legislación regional

(12) Para un estudio de la actividad financiera de las regiones, vide los Atti cit., págs. 379-479.

(13i) Referencias acerca del papel que la región desempeña en la ac. tuación de las descentralización, se contienen en nuestro trabajo La reforma de la Administractón pública en Iralia, publicado en el número 20 de la eRevista de Administración Públicas, págs. 121 y ss. 
y la inconsistencia de aquellas posturas doctrinales (14), a cuyo tenor la presencia de la Región incide sobre la estructura del Estado unitario para dar lugar a una nueva forma de Estado: el Estado Regional. Tal denominación puede corresponder al Estado ìtaliano sólo con un significado secundario y formal, para indicar... que junto a los Municipios y Provincias se encuentran, como entes territoriales de grado mayor, las Regiones (15).

\section{III}

Los razonamientos que preceden pudieran inducir. a pensar en una identidad entre la actividad administrativa desarrollada por las Regiones y la que ejercen los Municipios y Provincias; pero nada más lejos de la realidaid. La actividad administrativa regional presenta una serie de características que la distinguen de cualquier otra actividad de la misma especie. Son las siguientes: A) La autodisciplina normativa de la función administrativa regional; B) la rigurosa predeterminación de las materias que constituyen su objeto; C) la preeminencia de la Región sobre las Provincias y Municipios; y D) la singular estructura organizativa de la Región (16).

A) Hemos señalado ya la principal característica de la actividad administrativa regional: las disposiciones normativas que deben guiar esta actividad son en oran parte establecidas por el mismo ente, la Región.

B) Se habla de rigurosa predeterminación de las materias que constituyen el objeto de la actividad administrativa regional en el sentido de que, con la salvedad de la actividad administrativa delegada, el campo de accín de las Regiones sólo podrá ser modificado por una ley constitucional que modifique la Constitución o el correspondiente estatuto, segln que se trate de Regiones con régimen normal o especial, respectivamente. Esta predeterminación ddistingue la actividad administrativa que nos ocupa de la municipal o provincial, dado que los otros entes territoriales pueden desempeñar, junto a las funciones obligatorias, las funciones facultativas

(14) Vide, por ejemplo, Gurdo Lucatel. : Lo Stato regionale come nuova forma di Stato, en Atti cit., págs. 136-180.

(15) AMORTH : L'attivitd cit., pág. 311.

(16) Vide AMORTH : op. cit., cap. IV. 
que tengan por conveniente, con tal que su ejercicio no exorbite del respectivo ámbito territorial.

C) La Región no sólo actúa en un ámbito territorial donde se efectúa una acción de otros entes públicos, sino que, en parte, por la convergencia sobre materias que son también de la competencia de tales entes, en parte por la subordinación de los otros entes locales establecida por la Constitución, la preeminencia de la Región ofrece particularidades hasta ahora desconocidas por el ordenamiento jurídico italiano. Más adelante tendremos ocasión de estudiarlas, con más detenimiento (17).

D) Finalmente, la Región presenta una singular estructura organizativa. No se alude con ello a la existencia de dos Presidentes, uno de la Junta y otro del Consejo, que señalamos con anterioridad, puesto que esta nota es común a la Provincia, según el Derecho italiano, sino, en primer lugar, a la posibilidad de una actuación de los Asesores regionales como órganos externos, a diferencia de las Juntas municipales y provinciales, que operan sólo como órgano colegial, 'y, sobre todo, a la utilización de los órganos de las Provincias, Municipios y demás entes locales prevista por la Constitución (art. 118). Dicha utilización, con la que se pretende reducir al mínimo la burocracia regional, puede tener lugar mediante la delegación de funciones o el servicio de las oficinas de tales entes.

\section{IV}

Las Regiones, como entes autárquicos que son, se encuentran sometidas a los controles del Estado, de los que expondremos los principios generales contenidos en la Constitución y en la Ley de 10 de febrero de 1953, número 62. En la capital de cada Región se establece una Comisión de control, nombrada por Decreto del Presidente de la República, mediante propuesta del Presidente del Consejo de Ministros, de acuerdo con el Ministro del Interior. A dicha Comisión le corresponde tanto el control de legitimidad como el de mérito sobre las deliberaciones regionales. Toda deliberación regional que no sea de mera ejecución debe ser transmitida a la Comisión, que dentro de los veinte dias de haberla recibido puede pronunciar

(17) Vida luego apart. V. 
su anuiación por un vicio de legitimidad, o, en casos de control de mérito, puede invitar con requerimiento motivado al Consejo regional a que vuelva a examinarla. Si tal ocurre, o si dentro del término la Comisión comunica que no encuentra vicio de legitimidad, ni motivo para pedir el nuevo examen, las deliberaciones se convierten en ejecutivas.

Tratándose de control de legitimidad, la ejecutividad se suspende si, dentro del término referido, la Comisión de control pide aclaraciones o elementos integrativos de juicio a la Administración regional; pero la deliberación deviene igualmente ejecutîva si la Comisión no se pronuncia dentro de veinte días de haber recibido respuesta adecuada de la Región.

Para las deliberaciones sometidas exclusivamente al control de legitimidad, se consiente que sean $^{-}$declaradas inmediatamente ejecutables por específicas razones de urgencia, con el voto favorable de la mayoría absoluta de miembros asignados al organo deliberante. En tal caso, deben ser enviadas dentro de los tres días de la fecha en que fueron adoptadas y la Comisión puede pronunciar la anulación dentro de los diez días de la recepción.

Como controles extraordinarios sobre los órganos electivos, se prevén la disolución del Consejo regional y la sustituciớn de lạ Junta o del Presidente de la Región. El Consejo puede ser disuelto, con las formalidades que el artículo 126 de la Constitución y los artículos 52 y 53 de la Liey de 1953 establecen, cuando cumpla actos contrarios a la Constitución o graves violaciones de la ley; cuando, invitado por el Gobierno a sustituir a la Junta o al' Presidente que hayan realizado tales violaciones, no corresponda a tal invitacion; cuando, por dimisiones o por imposibilidad de formar una mayoría, el Consejo no se encuentre en situación de funcionar, y por razones de seguridad nacional (18).

(18) Para un estudio de los problemas relativos al control sobre la región, vide Mreze : La Regione cit,, cap. IV; VIRGa : La Regione cit., página 138 y ss. BENVENUTr : Il controllo mediante richiesta di riesame, en "Rivista Trimestrale di Diritto Pubblicos, 1954, pág. 377, citados por ZANOBINI : Corso cit., vol. III, pág. 204 , en nota. 
A lo largo de los capítulos anteriores hemos tenido ocasión de aludir a algunos de los principios que rigen las relaciones entre la Región y los demás entes locales. Hemos visto așí cb́mo, según el párrafo tercero del artículo 118 de la Constitución, la Región ejercitará normalmente sus funciones administrativas valiéndose de las oficinas de dichos entes. Pero esto no sería suficiente a preservarlos de los posibles ataques de una Región que pretendiese monopolizar, dentro de los límites de su territorio, el ejercicio de la actividad administrativa en las materias que se contienen en la amplia ènumeración del artículo 117. Por ello, el artículo 118, al señalar que la Región ejercerá las funciones administrativas en las mismas materias en que ejerce las legislativas, establece la salvedad de aquellas atribuídas a los entes locales por las leyes de la República. De esta suerte desaparece el peligro antes apuntado y la Constitución es consecuente con el principio fijado en su artículo $5 .^{\circ}$ de respetar y promover las autonomías locales y adaptar la legislación de la Republica a las exigencias de la descentralización. Un paso importante para conseguir esta meta es el que se da en el artículo 130 al estabiecer que la Región ejercitará el control sobre los actos de los entes locales; semejantes disposiciones se contienen en los estatutos de las Regiones con autonomía especial, si bien todavía no se han dictado las normas para su aplicación. Dichas normas se incluyen, en cambio, en la Ley de 10 de febrero de 1953, numero 62, en la que se establece un sistema de control similar al expuesto en el capítulo anterior.

Como es sabido, entre las facultades atribuídas a las Regiones por el artículo 117 se encuentra la de establecer el ordenamiento de las oficinas y de los entes dependientes de la Región y de cuanto se refiere a los términos de los entes locales. En igual sentido se expresan los estatutos de las Regiones especiales, aunque en ocasiones se establecen innovaciones que merecen les dediquemos especial atención. Nos referimos al artículc 15 del estatuto de la Región siciliana, en base al cual se suprimirían las Provincias como personas jurídicas autárquicas y como circunscripciones administrativas, con los Gobernadores civiles y con todos los órganos del Gobierno Civil. La norma no ha sido todavia aplicada, pero ha sido presentado a 
la Asamblea regional, que como se recordará es el nombre que en Sicilia recibe el Consejo regional, un proyecto de ley de delegación que, por las innovaciones que introduce, reviste singular interés.

Dicho proyecto (19) se refiere a la estructura de los entes locales, las funciones de los órganos directivos, la hacienda y los controles. Como base del nuevo ordenamiento administrativo de la Región se pone el Municipio, cuyo territorio debe coincidir con las actividades económicas y sociales de la población. Se suprimirán, en consecuencia, los Municipios con gran extensión territorial y escaso número de habitantes y los Municipios superpoblados con pequeño territorio.

En la distribución de las atribuciones correspondientes a los distintos órganos del Municipio, se intensifican las funciones ejecutivas de la Junta y se reservan al Consejo las deliberaciones que inciden sobre la dirección fundamental de la política administrativa y financiera del Municipio y los actos dispositivos de notable importancia.

En cuanto a la Hacienda municipal, el Gobierno de la Región actuará con la más amplia autonomía.

El problema del ente intermedio entre la Región y los Municipios se resuelve mediante el libre consorcio de Municipios, que tiene personalidad jurídica y es denominado Provincia. Los Municipios pueden elegir la Provincia a que pertenecer. Los criterios para la institución de la Provincia son los siguientes: a) mínimo número de Municipios y de población ; $b$ ) contigüidad de territorio entre los Municipios $; c$ ) afinidad en los intereses materiales y. morales prevalentes, puesta de manifiesto exclusivamente por los $\mathrm{Mu}$ nicipios que pretenden asociarse. También para la Provincia el Gobierno de la Región actuará con la más amplia autonomía financiera.

Para los controles, se establece en cada Provincia una Comisión de control sobre los actos de la Provincia, de los Municipios y de los entes locales en ella comprendidos. F1 control de legitimidad se realiza dentro de los veinte días de la recepción del acto a controlar, y el de méritos se ejercita, en pocos casos, mediante requerimiento motivado a los entes deliberantes de reexaminar su deliberación.

- (19) Vide SALEmI : L'attivitd amministrativa della Regione Siciliana, en Atti cit., págs. 249-263, en especial el núm. 9. 


\section{VI}

Un examen de la actividad administrativa de las Regiones, siquiera sea en la forma somera que lo hemos realizado, quedaría incompleto si no dedicásemos unas líneas a los resultados de la referida actividad. El problema ha sido estudiado por Benvenuti, que, a tal fin, distribuye en tres grapos las materias atribuídas a la competencia regional. En el primer grupo incluye aquellas materias en que tienen competencia tanto las Regiones con estatuto normal, como las Regiones con estatuto especial ; materias que denomina comunes. Como materias especiales considera aquellas sobre las que tienen competencia todas las Regiones con estatuto especial, pero no las de estatuto normal ; finalmente, el tercer grupo se encuentra integrado por las materias particulares, es decir, aquellas sobre las que tienen competencia. alguna de las Regiones con estatuto especial, pero no las demás.

Pues bien, el grupo que más ha llamado la atención de las Regiones hạ sido el de las materias particulares, en el que se ha legislado con gran intensidad, verificándose, por tanto, el tránsito de la competencia administrativa del Estado a la de las Regiones. Ello demuestra, en la opinión del autor citado, cla aptitud de la administración regional de los intereses públicos para proveer a aquellas necesidades que le son más especialmente peculiares, en lugar del Estado, necesariamente lejano y comprobadamente ausente (20). También las materias del segundo grupo han sido objeto de singular cuidado, especialmente en cuanto se refiere a las obras encaminadas a la protección de la agricultura; a las minas; a la producción y distribución de la energía eléctrica; a la industria y el comercio; a los seguros sociales, y a la instrucción elemental. En las materias comunes la mayor actividad se ha desarrollado con relación al turismo; las obras públicas; la caza y pesca; la artesanía y, sobre todo, respecto de la agricultura. Quedan, por tanto, más desatendidas, entre las materias especiales, la expropiación forzosa ; las bellas artes; los servicios publicos, y los espectáculos; $\mathbf{Y}$,

(20) Risultati e prospettive dell'attivitd regionale, en Atti cit., páginas $473-488$. 
entre las comunes, la policía local ; las ferias y mercados; la beneficencia pública ; los museos y bibliotecas ; los tránvías, y los transportes por carretera. Es decir, aquellas materias de menos interés o de carácter técnico.

Visto cuanto precede, podemos resaltar, con Benvenuti, el hecho de que la mayor sensibilidad de la Región se ba sentido atraída por los problemas más propiamente económicos y sociales de là vịda de las comunidades locales. Lo que, desde otro punto de vista, significa aque en la solución de estọs problemas la actividad del Estado era manifiestamente insuficiente, es decir, incapaz de afrontarlos en forma exacta $y$ completa, precisamente por defecto de sensibilidad local o, lo que es igual, de un exacto conocimiento de los distịntos problemas territoriales delimitadoss.

\section{Rafaei, Entrena Cuesta}

Doctor en Derecho por la Universidad de Bolonia. 